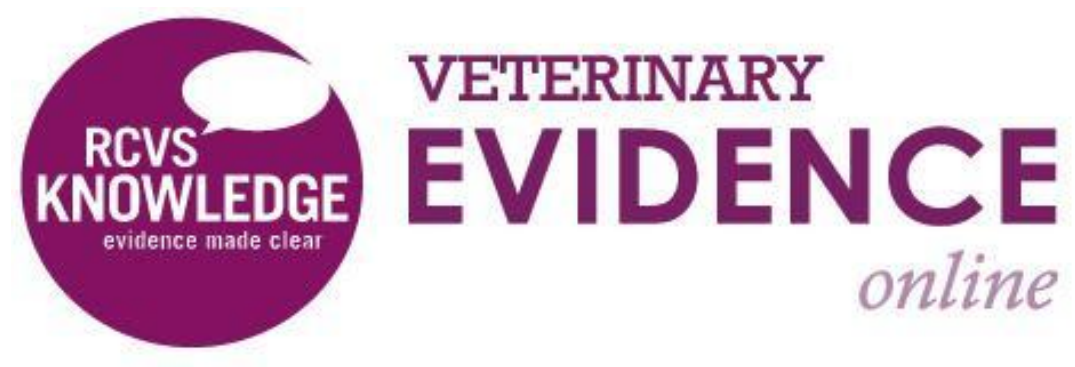

\title{
Net-bottom Cage Inserts for Water Bird Casualties
}

Jackie Belle RVN CertSAN CertFN CertVNES DipCABT DipESM Dip BR DipEnDv PTLLS BSC MSC ${ }^{1^{*}}$

\footnotetext{
${ }^{1}$ RCVS Knowledge, 62-64 Horseferry Road, London, SW1P 2AF

Corresponding Author (Jackie@rcvsknowledge.org))
}

ISSN: 2396-9776

Published: 31 Oct 2017

in: Vol 2, Issue 4

DOI: http://dx.doi.org/10.18849/ve.v2i4.66

Reviewed by: Jonathan cracknell (BVMS CertVA CertZooMed MRCVS), Simon Girling (BVMS (Hons) DZooMed DipECZM (ZHM) CBiol FRSB EurProBiol FRCVS) and John Chitty (BVetMed CertZooMed CBiol MRSB MRCVS) 
My Bright Idea is a net-bottomed cage insert, which is used to support pelagic avian casualties. The idea was designed and modified by the International Bird Rescue in California (Bird Rescue).

When nursing wildlife casualties there are often challenges in converting existing small animal hospital accommodation to provide appropriate and suitable facilities for triaging wildlife patients - before they are transferred to a wildlife facility.

As some aquatic birds, such as loons and grebes, are unable to stand on a terrestrial substrates, traditional cat and dog cages are much less suitable for their care. At the Bird Rescue facility, aquatic species such as the divers, grebes, guillemots and sea ducks were all housed in net-bottomed cages.

Grebes and divers particularly struggle in captivity as they have long, heavy bodies with legs at the rear (which act as rudders in the water), when on land they move with little jumps and slides. These types of aquatic birds do not spend time on land and are only ever on land during nesting since moving is so difficult on the hard substrate. If these birds are housed in net-bottomed caging they are less prone to pressure sores, injury to their legs and feather rot (prevents urates from building up on the tail feathers).

\section{Net-bottomed cage design}

In the net-bottomed cage design, the floor is made of a PVC pipe frame covered with half-inch knotless shrimp seine (black or white) which is stretched taught then fastened over screws placed on the outside of the frame. The netting provides an even weight distribution and allows faeces and urates to drop through onto newspaper on the cage floor. A heat pad can also be placed above the newspaper to increase the temperature of hypothermic animals. The gap between the PVC flooring and cage bottom allows air to circulate and ventilate the cage.

Cages were left prepared (Photo 1), with a towel which is used to hang on the inside of the cage door to decrease stress by limiting visual stimulus.



Photo 1: Hospital cage showing raised net-bottom insert 
We also converted plastic carrier cages for use when transporting pelagic bird. These carriers were converted by placing a net over a PVC frame at the base of the box to prevent injury during transport. The inserts are also easily removed to convert back to a normal pet carrier.

These can also be used as haul-outs over water (Photo 2 and 3).

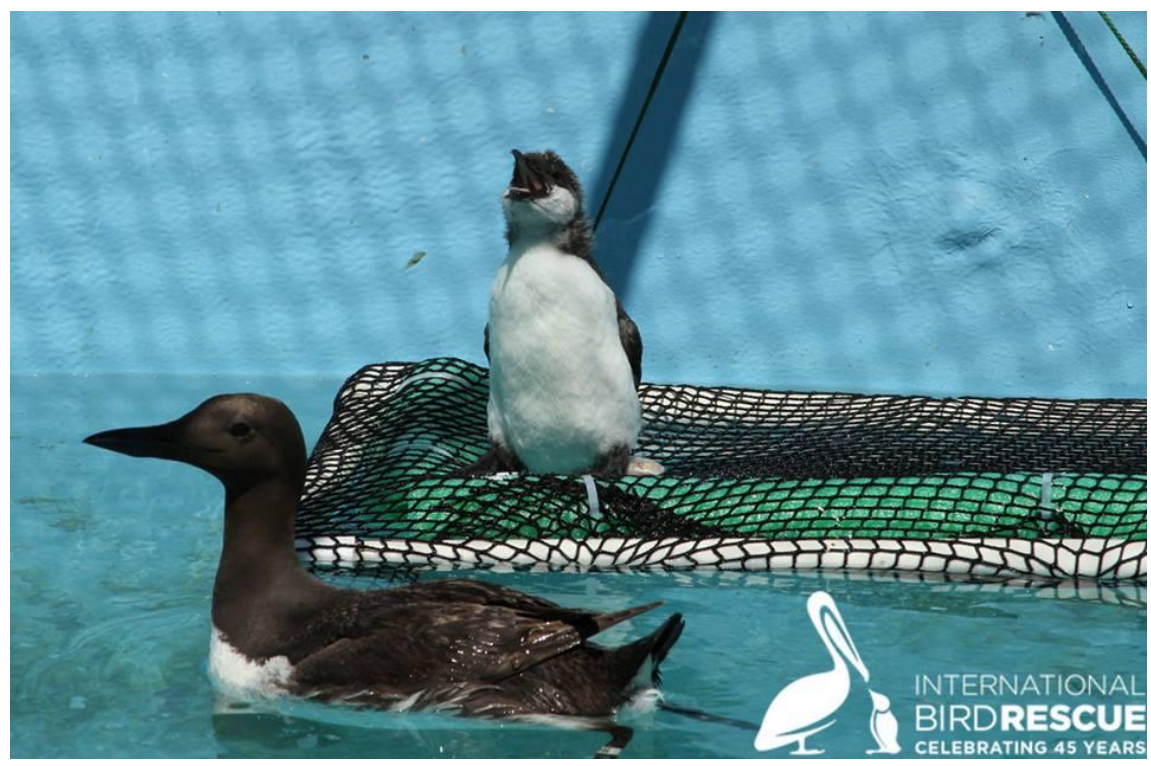

Photo 2: Murres (guillemots) on haul-out raft made with PVC and netting.

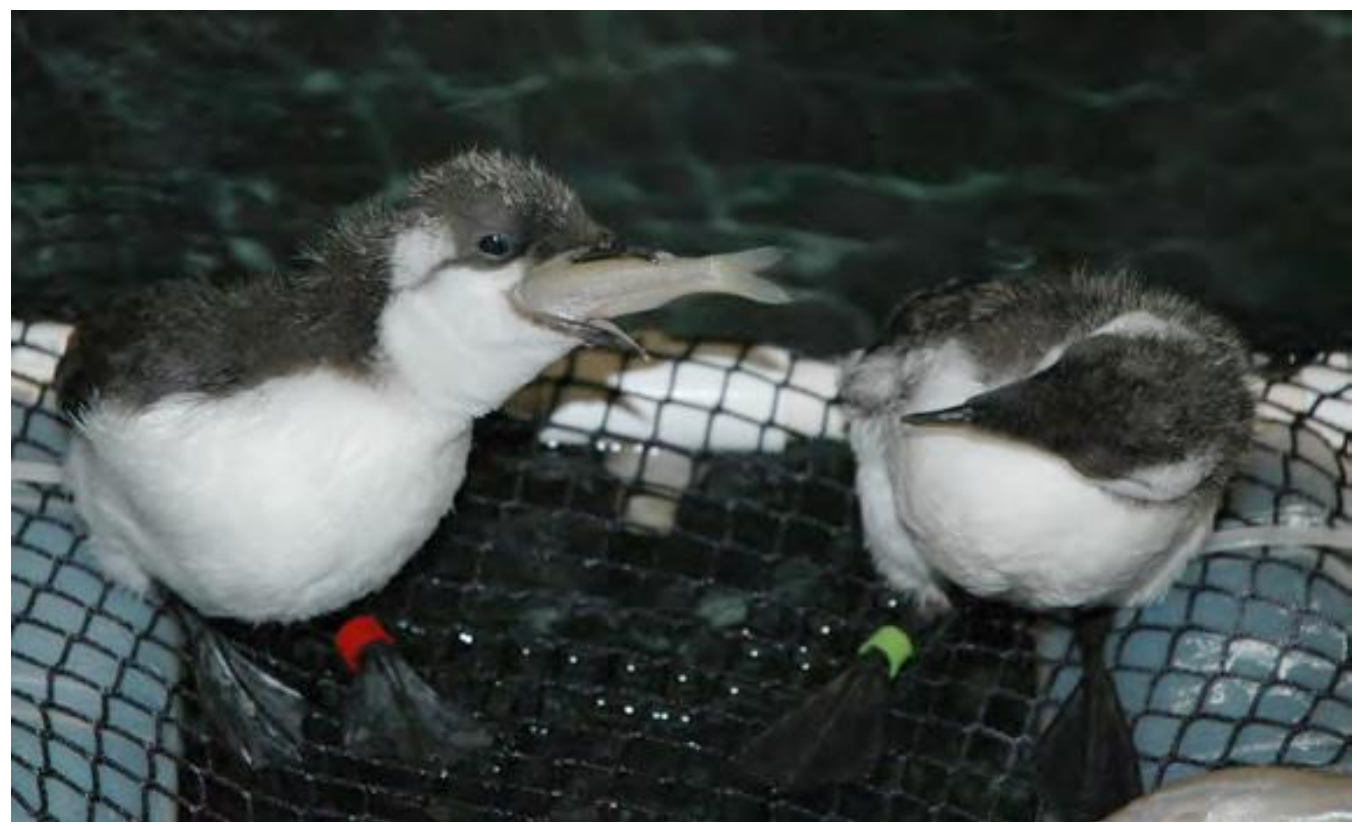

Photo 3: Bird Rescue image: Common murres feeding on net-bottomed cage insert 


\section{Net-bottom cage inserts}

\section{Materials}

- PVC piping and elbows (1.5")

- Nylon netting $\left(1 / 4^{\prime \prime}-1 / 2 "\right)$

- Wood screws

\section{Instructions}

- Make a rectangle frame out of PVC piping and elbows

- Be sure to measure the desired size of the insert by the "outside" measurement of the frame

- Attach screws to the underside of the frame

- Leave the screw heads with approximately $1 / 8$ " exposed, this creates a 'peg' to attach the nylon netting

- Space the screws evenly, approximately 3"-4" apart

- Stretch nylon netting tightly over the frame and secure by looping the netting over the screw heads (peg).

The specifications of the netting that Bird Rescue uses for the net-bottomed pens are at least $1 / 4$ " up to $1 / 2$ " knotless nylon netting. This size will stop small birds falling through and evenly distribute the weight of small and medium sized birds (grebe, duck). The photo below of an albatross also demonstrates the diameter of the mesh required.

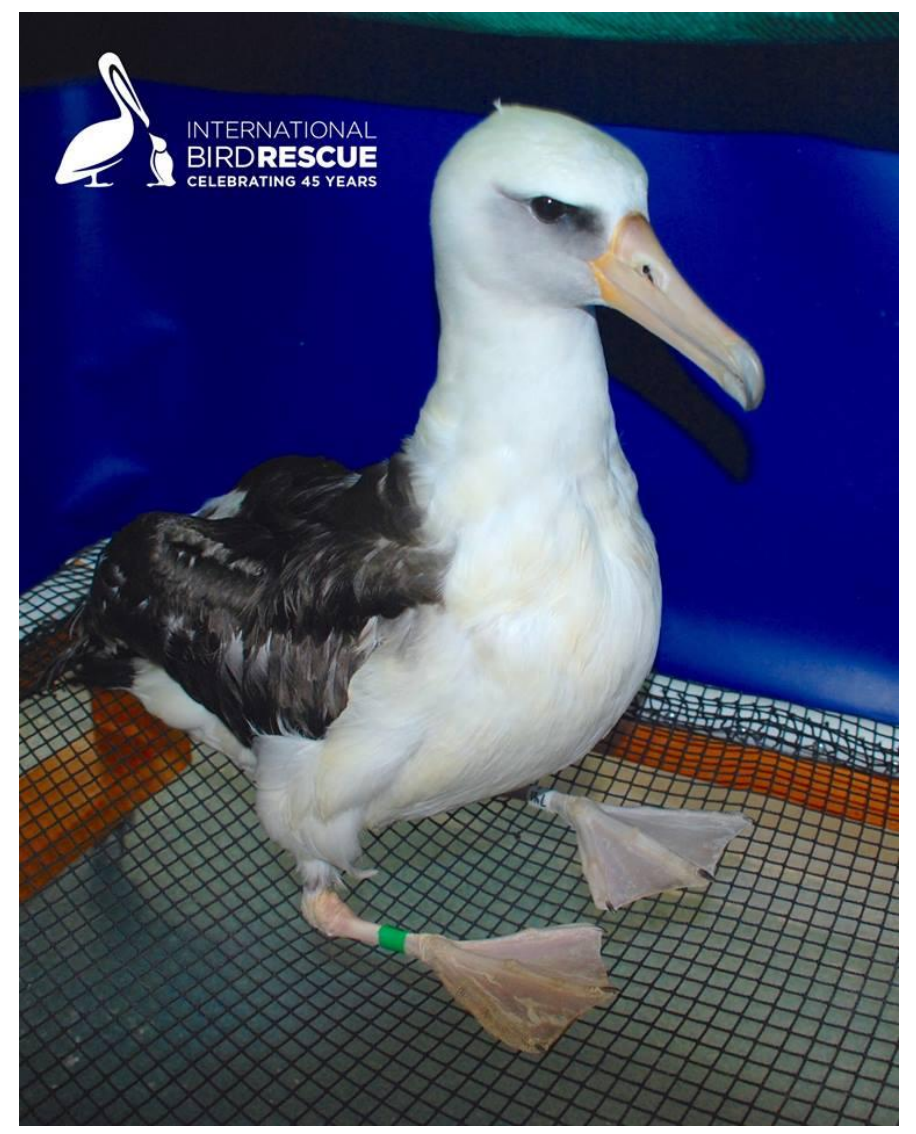

Photo 4: Laysan albatross in drying cage following wash to remove oil 
If the nylon mesh becomes damaged or torn it is best to replace the entire net bottom rather than trying to repair a damaged area. A break in the mesh could lead to entanglement or foot abrasion and alter the supportive surface of the netting insert.

Contact International Bird Rescue for further information: https://www.bird-rescue.org/

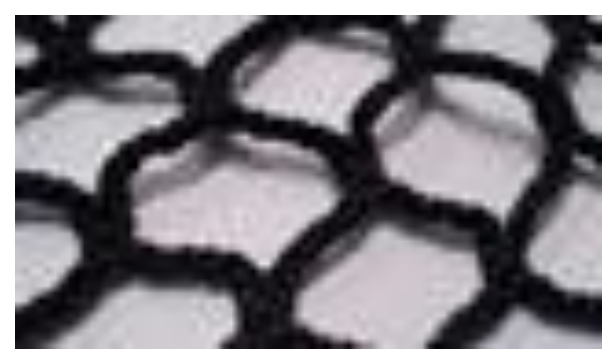

Photo 5: Example netting $\left(1 / 4{ }^{\prime \prime}-1 / 2{ }^{\prime \prime}\right)$

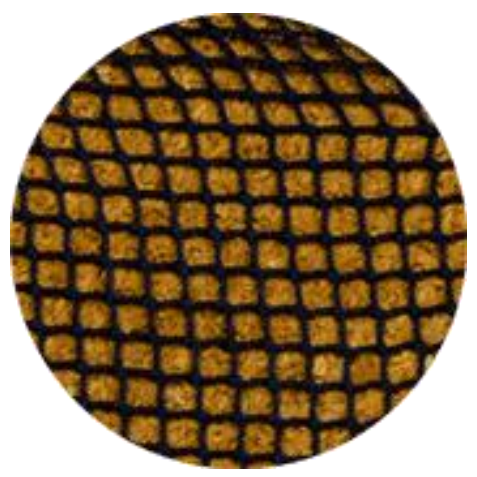

Photo 6: Example netting $\left(1 / 4{ }^{\prime \prime}-1 / 2{ }^{\prime \prime}\right)$

\section{Netting Suppliers in USA}

The product is referred to as a Delta or Ace style Netting Christenson Net Works

4074 Sucia Dr.

Ferndale, WA 98248

\section{CONFLICT OF INTEREST}

The author delares no conflict of interest.

\section{REFERENCES}

NA 


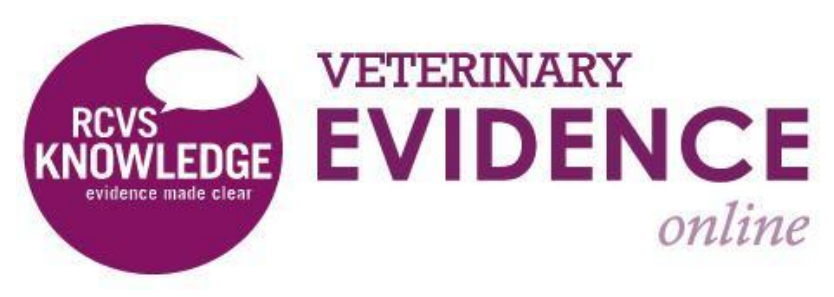

\section{Intellectual Property Rights}

Authors of Articles submitted to RCVS Knowledge for publication will retain copyright

in their work, and will be required to grant to RCVS Knowledge a non-exclusive

license of the rights of copyright in the materials including but not limited to the right to publish, re-publish, transmit, sell, distribute and otherwise use the materials in all languages and all media throughout the world, and to license or permit others to do

so.

Veterinary Evidence and EBVM Network are RCVS Knowledge initiatives. For more information please contact us at editor@veterinaryevidence.org.

RCVS Knowledge is the independent charity associated with the Royal College of Veterinary Surgeons (RCVS). Our ambition is to become a global intermediary for evidence based veterinary knowledge by providing access to information that is of immediate value to practicing veterinary professionals and directly contributes to evidence based clinical decision-making.

\section{www.veterinaryevidence.org}

RCVS Knowledge is a registered Charity No. 230886. Registered as a Company limited by guarantee in England and Wales No. 598443.

Registered Office:

Belgravia House

62-64 Horseferry Road

London SW1P 2AF 